\title{
DESENVOLVIMENTO DE FILMES ORODISPERSÍVEIS BIOPOLIMÉRICOS À BASE DE AMIDO, GOMA XANTANA E GELATINA
}

\author{
Jéssica Fernanda Pereira* \\ Beatriz Marjorie Marim ${ }^{* *}$ \\ Suzana Mali ${ }^{* k+}$
}

\begin{abstract}
RESUMO: Recentemente tem aumentado o interesse no desenvolvimento de novos produtos obtidos a partir de matérias-primas de fonte renovável em todos os setores industriais, incluindo a indústria farmacêutica. 0 presente trabalho teve como objetivo produzir filmes orodispersíveis (ODFs) à base de amido de mandioca, gelatina e goma xantana, pelo processo de casting, assim como caracterizá-Ios quanto à sua microestrutura, solubilidade, grau de intumescimento, permeabilidade ao vapor de água e propriedades mecânicas. Foi empregado delineamento experimental de misturas ternárias para se avaliar o efeito dos três biopolímeros sobre as propriedades dos filmes produzidos. Os filmes à base de gelatina pura e a mistura binária entre amido e gelatina se mostraram às alternativas mais promissoras para serem usadas como matrizes poliméricas para a liberação de princípios ativos na cavidade bucal, uma vez que estes apresentaram os melhores resultados de solubilidade e as melhores propriedades mecânicas.
\end{abstract}

PALAVRAS-CHAVE: Cavidade bucal; Fonte renovável; Biopolímeros.

\section{DEVELOPMENT OF STARCH, XANTHAN AND GELATIN BASED BIOPOLYMER ORODISPERSIVE FILMS}

\begin{abstract}
Interest in the development of new products from renewable prime matter sources in all types of industries, including the pharmaceutical one, is on the increase. Current assay produces oro-dispersible films (ODFs) based on manioc starch, gelatin and xanthan gum by the casting process. Microstructure, solubility, intumescence degree, water vapor permeability and mechanic properties are also characterized. Experimental design comprised ternary mixtures to evaluate the effect of three biopolymers on the films produced. Pure gelatin-based films and starch-gelatin binary mixture films were the most promising alternatives for polymer matrixes for the release of the active principle in the mouth cavity. In fact, they were the best results in solubility and the best mechanical properties obtained.
\end{abstract}

KEYWORDS: Buccal Cavity; Renewable source; Biopolymers.

\section{INTRODUÇÃo}

0 interesse mundial no emprego de biopolímeros aumentou nos últimos anos em função do desejo de se substituir os materiais poliméricos obtidos a partir de recursos fósseis por matérias-primas advindas de fontes renováveis, mas também como consequência dos avanços inovadores alcançados na biotecnologia (GALIANO et al., 2018).

0 desenvolvimento de filmes biopolímeros tem sido amplamente explorado com diferentes propostas de utilização, como em embalagens de alimentos (LUCENA et al., 2017), revestimentos comestíveis (ROMIO et al.,

\footnotetext{
* Mestranda em Biotecnologia na Universidade Estadual de Londrina (UEL), Brasil.

** Mestranda em Biotecnologia na Universidade Estadual de Londrina (UEL), Brasil.

${ }^{* * *}$ Doutora em Ciência de Alimentos. Docente Associada do Departamento de Bioquímica e Biotecnologia e no Programa de Mestrado e Doutorado em Biotecnologia da Universidade Estadual de Londrina(UEL). Brasil. E-mail: smali@uel.br.
} 
2017), recobrimentos de sementes (VERCELHEZE et al., 2019), e também na área farmacêutica, inicialmente para 0 recobrimento de comprimidos (VILLANOVA; OREFICE; CUNHA, 2010), como objetivo para proteger o fármaco de condições agressivas do meio ambiente, tais como luz e umidade, e do microambiente fisiológico, além de aprimorar aspectos sensoriais como aparência e sabor, e auxiliar e/ou modular a liberação do fármaco.

Mais recentemente, 0 desenvolvimento de filmes orodispersíveis (ODFs) tem chamado a atenção do setor industrial farmacêutico, e as primeiras pesquisas com esses filmes foram no combate ao mau hálito bucal, e a evolução desses filmes tem se dado através da incorporação de fármacos com as mais diversas aplicações (BORGES et al., 2015).

Os ODFs podem ser considerados como uma alternativa às formas farmacêuticas orais convencionais, e consistem em películas ultrafinas, que quando colocadas sobre a língua são hidratadas pela saliva e rapidamente se aderem ao local de aplicação, onde 0 fármaco é liberado e absorvido pela mucosa bucal (MANDEEP; RANA; NIMRATA, 2013; PATEL et al., 2012). A absorção de fármacos via mucosa bucal evita a exposição ao baixo pH gástrico, ação de proteases e degradação pelo efeito de primeira passagem no fígado (TEDESCO; MONACO-LOURENÇO; CARVALHO, 2016). Não há necessidade de administração dos ODFs com água, uma vez que são deglutidos, e quando administrados dificilmente podem ser removidos da cavidade bucal (CASTR0 et al., 2017). Além disso, são produtos de fácil transporte e manuseio (PALLAVl; SHRIVASTAVA, 2014; PRAJAPATI; RATNAKAR, 2009).

Para a produção dos ODFs geralmente são utilizados polímeros hidrofílicos (solúveis em água) que, quando em contato com a saliva se dissolvem completamente na boca. Além disso, existem várias características que podem ser controladas com a escolha do polímero, como 0 tempo de desintegração e dissolução do fármaco, a resistência mecânica e elasticidade, entre outros fatores. Por isso a escolha dos polímeros é um passo crítico da pesquisa e pode variar haja vista o perfil do produto desejado (MANDEEP; RANA; NIMRATA, 2013; PALLAVI; SHRIVASTAVA, 2014).
Os polímeros utilizados para a produção dos filmes também devem apresentar algumas características, tais como serem atóxicos, não irritantes, apresentarem ausência de gosto e não retardarem o tempo de desintegração dos filmes. Diante disto, 0 amido de mandioca, a gelatina e a goma xantana são polímeros que apresentam propriedades interessantes para uso na produção de ODFs (MALI et al., 2005; PRAJAPATI; RATNAKAR, 2009; SUEIRO, 2018; TEDESCO; MONACO-LOURENÇO; CARVALHO, 2016; VERCELHEZE et al., 2019).

A gelatina é uma proteína obtida através da hidrólise ácida ou alcalina do colágeno, isolado a partir de pele, ossos ou tecido conjuntivo de animais. É facilmente solúvel em água sob temperatura média de $40^{\circ} \mathrm{C}$ e vem sendo amplamente explorada em estudos que envolvem a produção de filmes biodegradáveis e/ ou comestíveis (BORGES et al., 2015; SU; WANG, 2015; VERCELHEZE et al., 2019).

0 amido de mandioca é um homopolissacarídeo de glicose, com caráter hidrofílico, obtidos de diferentes fontes vegetais e de baixo custo (PARK et al., 2017), porém filmes de amido são frágeis e quebradiços, por isso é importante 0 estudo de misturas poliméricas que possam melhorar o seu desempenho (PRAJAPATI; RATNAKAR, 2009).

A goma xantana é um polissacarídeo hidrossolúvel de grande interesse na indústria farmacêutica e de alimentos, sendo sintetizado por uma bactéria fitopatogênica do gênero Xanthomonas. 0 interesse pela goma xantana se deve às suas propriedades físico-químicas, que permitem a formação de soluções viscosas a baixas concentrações, estabilidade em ampla faixa de $\mathrm{pH}$ e temperatura, e a viscosidade que é pouco afetada na presença de sais (VILLANOVA; OREFICE; CUNHA, 2010).

Além dos polímeros, a formulação dos ODFs deve conter outros aditivos, como plastificantes, que conferem a flexibilidade ao filme reduzindo sua fragilidade, agentes estimulantes de saliva, pois a quantidade de saliva a influencia no tempo de desintegração do filme (BORGES et al., 2015), edulcorantes, aromatizantes, conservantes e corantes 
que melhoram a aceitação sensorial dos filmes (MANDEEP; RANA; NIMRATA, 2013; VILLANOVA; OREFICE; CUNHA, 2010).

0 objetivo desse trabalho foi a produção de filmes orodispersíveis à base de amido de mandioca, gelatina e goma xantana, pelo processo de casting. Os filmes foram caracterizados quanto à sua microestrutura, solubilidade, grau de intumescimento, permeabilidade ao vapor de água e propriedades mecânicas.

\section{METODOLOGIA}

\subsection{MATERIAL}

0 amido de mandioca empregado (Yoki Paranavaí-PR) foi obtido no mercado local. Foi empregada gelatina da marca BIOTEC (São Paulo, Brasil). A goma xantana foi obtida da Synth (São Paulo, Brasil).

\subsection{PREPARO DOS FILMES ORODISPERSÍVEIS}

Os filmes foram produzidos por casting, técnica em que a solução filmogênica $(1,50 \%$ m/v) foi preparada em meio aquoso, colocada sobre um suporte para desidratação, e após a evaporação do solvente 0 filme foi removido por destacamento. Foram elaboradas sete formulações de revestimentos empregando-se delineamento experimental de misturas ternárias (Tabela 1), variando-se 0 teor dos polímeros empregados, que foram amido de mandioca, gelatina e goma xantana. Foram preparadas soluções filmogênicas a partir das formulações descritas na Tabela 1.

Em todas as formulações empregou-se 0,3 $\mathrm{g}$ de glicerol $(0,3 \mathrm{~g} / 100 \mathrm{~mL}$ de solução) como plastificante. Para 0 preparo dos revestimentos, a gelatina foi hidratada em água destilada à temperatura ambiente por 30 min e depois solubilizada a $55^{\circ} \mathrm{C}$ por 30 min. 0 amido de mandioca foi aquecido a $90^{\circ} \mathrm{C}$ por 10 min. A goma xantana foi hidratada em água destilada à temperatura ambiente por $12 \mathrm{~h}$ e depois solubilizada a $90^{\circ} \mathrm{C}$ por $30 \mathrm{~min}$. Por fim, para o preparo das misturas, os componentes de cada formulação foram misturados nas proporções pré-estabelecidas e agitados continuamente em banho-maria (Marconi MA 127) a $90^{\circ} \mathrm{C}$ por 30 min. As soluções filmogênicas $(20 \mathrm{~g})$ foram secas em placas de acrílico em estufa (Marconi modelo MA 035, São Paulo) com circulação de ar a $30^{\circ} \mathrm{C}$ por $24 \mathrm{~h}$.

Tabela 1. Formulações das soluções filmogênicas empregadas para o preparo dos filmes orodispersíveis

\begin{tabular}{lcccccc}
\hline & \multicolumn{3}{c}{ Componentes originais (\%) } & \multicolumn{3}{c}{ Valores codificados } \\
\hline Formulaçao & $\begin{array}{c}\text { Amido } \\
\left(X_{1}\right)\end{array}$ & $\begin{array}{c}\text { Gelatina } \\
\left(X_{2}\right)\end{array}$ & $\begin{array}{c}\text { Goma } \\
\text { xantana } \\
\left(X_{3}\right)\end{array}$ & $X_{1}$ & $X_{2}$ & $X_{3}$ \\
\hline F1 & 1,50 & - & - & 1 & 0 & 0 \\
F2 & - & 1,50 & - & 0 & 1 & 0 \\
F3 & - & - & 1,50 & 0 & 0 & 1 \\
F4 & 0,75 & 0,75 & - & 0,5 & 0,5 & 0 \\
F5 & 0,75 & - & 0,75 & 0,5 & 0 & 0,5 \\
F6 & - & 0,75 & 0,75 & 0 & 0,5 & 0,5 \\
F7 & 0,50 & 0,50 & 0,50 & 0,33 & 0,33 & 0,33 \\
\hline
\end{tabular}

\subsection{CARACTERIZAÇÃO DOS FILMES}

Antes das análises, as amostras dos filmes foram condicionadas em umidade relativa de $58 \%$ a 25C em estufa incubadora para BOD (Marconi - São Paulo).

A espessura dos filmes foi determinada utilizando um micrômetro manual Mitutoyo (São Paulo - Brasil) com resolução de $1 \mu \mathrm{m}$. Foram realizadas medições em três pontos distintos de cada corpo de prova, e a espessura foi tomada como a média aritmética das medições em seis corpos de prova de cada formulação de filme.

$0 \mathrm{pH}$ dos filmes foi determinado diluindo-se $1,0 \mathrm{~g}$ de cada amostra, em triplicata, em 9,0 mL de água destilada, e a mistura foi mantida sob agitação por 01 hora. As determinações de $\mathrm{pH}$ foram realizadas em potenciômetro (Digimed DM20, São Paulo - Brasil) sob temperatura ambiente. Todos os ensaios foram 
realizados em triplicata.

A análise da microestrutura dos filmes foi realizada por microscopia eletrônica de varredura (MEV) em microscópio eletrônico de varredura FEI Quanta 200 (Oregon - EUA). As amostras foram imersas em nitrogênio líquido para congelamento rápido, fraturadas com auxílio de pinças e secas em cloreto de cálcio $\left(\mathrm{CaCl}_{2}\right)$ por uma semana. As amostras secas foram revestidas de ouro por um Sputter Coater BAL-TEC SCD 050. As amostras foram analisadas em microscópio eletrônico de varredura do Laboratório de Microanálise e Microscopia Eletrônica da UEL.

A permeabilidade ao vapor de água (PVA) foi determinada gravimetricamente, de acordo com as normas (ASTM E96-00) da American Society for Testing and Material (2000a). Cada amostra de filme foi colocada em uma célula de permeabilidade selada, com abertura circular de $60 \mathrm{~mm}$, de modo a garantir que a transferência de vapor de água ocorresse exclusivamente através do filme. As células foram parcialmente preenchidas com $\mathrm{CaCl}_{2}$ de modo a se obter $0 \%$ de umidade relativa (UR) em seu interior e 0 conjunto (célula + filme) foi colocado em dessecador a $25^{\circ} \mathrm{C}$, este previamente preparado com solução saturada de $\mathrm{NaCl}$ para proporcionar ambiente com $75 \%$ UR. Após um período de equilíbrio de $3 \mathrm{~h}$, as células foram pesadas em intervalos regulares até obtenção de uma taxa constante de ganho de peso. 0 aumento do peso $(\mathrm{g})$ foi registrado e plotado em gráfico em função do tempo (s). 0 coeficiente angular de cada reta foi calculado pela regressão linear no regime constante $\left(r^{2}\right)$ e a PVA foi calculada de acordo com a equação 1:

$$
P V A=\frac{g}{t} \times \frac{\theta}{A \Delta P} P V A=\frac{g}{t} \times \frac{\theta}{A \Delta P}
$$

$g / t(g r a m a s / s)=$ coeficiente angular da reta

$A$ = área de permeação $\left(m^{2}\right)$

$e=$ espessura média dos filmes $(m)^{\star}$

$P=$ diferença de pressão de vapor de água $(\mathrm{KPa})$

As propriedades mecânicas de tração foram analisadas em texturômetro Stable Micro Systems modelo TA.TX2i (Surrey - Inglaterra) de acordo com as normas (ASTM D882-91) da American Society for Testing and Material (2000b). Os corpos de prova $(25 \times 100 \mathrm{~mm})$ foram cortados e ajustados entre as garras pneumáticas do equipamento. A distância entre as garras foi de $50 \mathrm{~mm}$ e a velocidade de tração de $500 \mathrm{~mm} / \mathrm{min}$. Foram determinadas as seguintes propriedades: resistência máxima à tração $(\mathrm{MPa})$ e a elongação na ruptura (\%).

A solubilidade e o grau de intumescimento (Gl) foram determinados simultaneamente pelo método gravimétrico, utilizando-se um dispositivo cilíndrico previamente pesado, contendo em uma das extremidades uma malha com abertura nominal de 138 x $75 \mu \mathrm{m}$, de acordo com Sueiro (2018). Corpos de prova $(2 \mathrm{~cm} \times 2 \mathrm{~cm})$ de cada formulação foram cuidadosamente pesados e colocados no dispositivo e, em seguida, estes foram imersos em $10 \mathrm{~mL}$ de solução tampão fosfato ( $\mathrm{pH} 7,0)$. Em tempos pré-determinados $(0,5,1,2,3$ e 4 min), os dispositivos foram retirados da solução, e o excesso de tampão foi retirado com auxílio de papel filtro, e os dispositivos foram pesados em balança analítica (Marte AY220, São Paulo), e a partir do valor obtido foi calculado o Gl de cada amostra em relação ao seu peso seco inicial. Em seguida, os filmes foram secos em estufa de circulação de ar com temperatura controlada de $25^{\circ} \mathrm{C}$ por 24 horas. Ao fim desse período os filmes foram pesados novamente, $\mathrm{e}$ então foi calculada a porcentagem de solubilização de cada amostra.

\subsection{ANÁLISE ESTATÍSTICA}

Para a análise de alguns dos resultados de caracterização dos filmes foram obtidos modelos matemáticos quadráticos (Equação 2) para as respostas de permeabilidade ao vapor de água (PVA) e propriedades mecânicas de tração (resistência máxima à tração (RMT) e elongação) empregando-se o software Statistica 7.0 (Statsoft, Oklahoma, USA):

$Y=b_{1} X_{1}+b_{2} X_{2}+b_{3} X_{3}+b_{12} X_{1} X_{2}+b_{13} X_{1} X_{3}+b_{23} X_{2} X_{3}(2)$ em que Y é a variável dependente ou resposta, 
$b_{1}, b_{2}, b_{3}, b_{12}, b_{13}, b_{23}$ são os parâmetros estimados para cada termo linear e de interação obtidos através dos modelos em que as variáveis foram amido de mandioca $\left(X_{1}\right)$, gelatina $\left(X_{2}\right)$, e goma xantana $\left(X_{3}\right)$. Gráficos de contorno foram obtidos para a análise dos efeitos das variáveis estudadas.

Os dados obtidos para o solubilidade e grau de intumescimento (GI) das amostras em função do tempo foram submetidos ao teste de Tukey de comparação de médias $(p \leq 0,05)$ empregando-se 0 software Statistica 7.0 (Statsoft, Oklahoma, USA).

\section{RESULTADOS E DISCUSSÃO}

Todas as formulações levaram à formação de filmes inteiros, contínuos, sem poros ou rachaduras, e fáceis de manusear. Os filmes contendo goma xantana apresentaram coloração amarelada, enquanto os filmes de amido e gelatina se mostraram translúcidos. A espessura dos filmes variou de 40 a $56 \mathrm{~mm}$ e $0 \mathrm{pH}$ dos filmes variou de 6,0 a 7,2, com valores próximos da neutralidade, o que é uma característica interessante para a incorporação de fármacos, o que não afetaria a estabilidade pelo fármaco ser exposto a valores extremos de pH.

Na Figura 1 estão apresentadas as imagens obtidas por microscopia eletrônica de varredura da superfície dos filmes, no geral, as superfícies das formulações se mostraram homogêneas com a ausência de porosidade ou rachaduras.

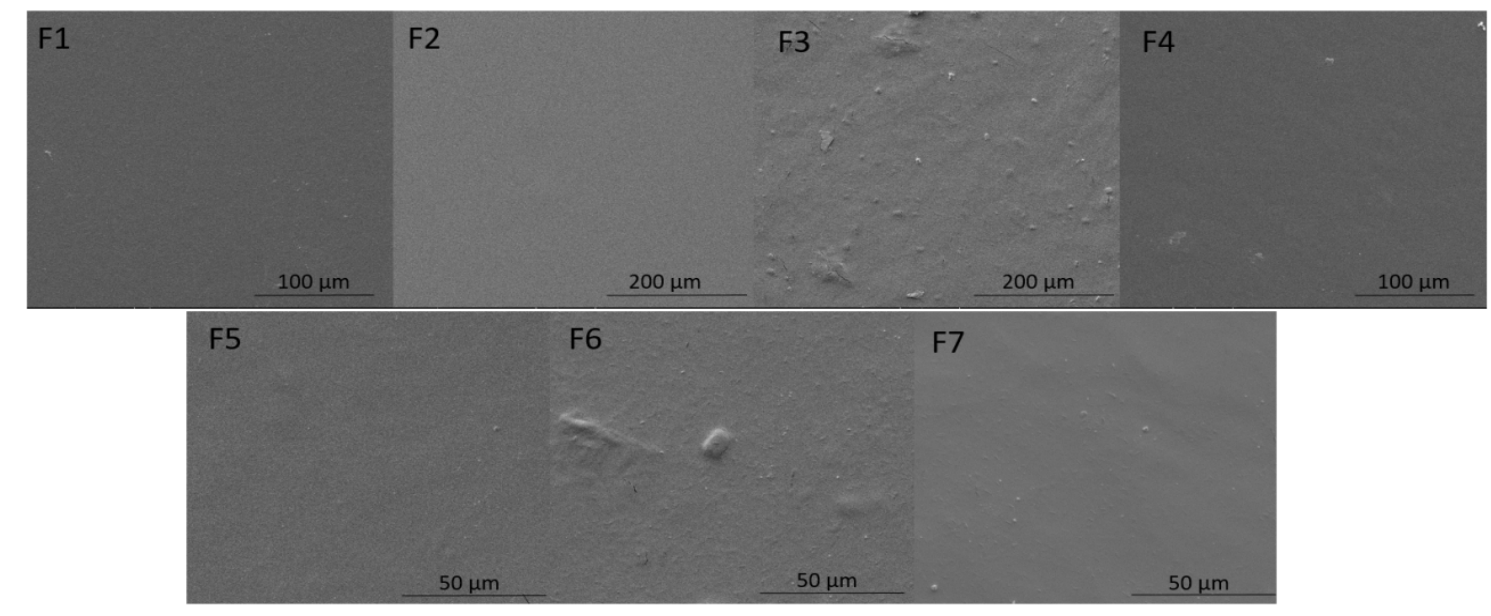

Figura 1. Microscopia eletrônica de varredura da superfície dos filmes

F1 (amido puro), F2 (gelatina pura), F3 (xantana pura), F4 (mistura binária amido e gelatina), F5 (mistura binária amido e xantana), F6 (mistura binária gelatina e xantana) e F7 (mistura ternária amido, gelatina e xantana)

Os resultados de permeabilidade ao vapor de água e propriedades mecânicas dos filmes estão apresentados na Tabela 2, e os modelos matemáticos obtidos para estas respostas estão apresentados na Tabela 3. Pode-se observar que para a permeabilidade ao vapor de água (PVA), os três biopolímeros apresentaram efeitos positivos e significativos (Tabela 3) sobre esta resposta, e maior efeito foi observado para a goma xantana $\left(X_{3}\right)$, no entanto o modelo obtido não foi significativo $(p=0,3632)$. Na Figura 2 pode-se observar que os maiores valores de PVA foram observados nos maiores teores de goma xantana, e o filme com maior PVA foi a formulação F3, produzido com a goma xantana pura (Tabela 2).

Os valores de resistência máxima a tração e a elongação apresentaram grande variação entre os filmes (Tabela 2). Estas propriedades estão diretamente relacionadas com as macromoléculas utilizadas para 
a formação da matriz polimérica (MALI et al., 2005). Entre os filmes preparados com os polímeros puros, os produzidos somente com gelatina (F2) apresentaram a maior RMT, enquanto os produzidos somente com a goma xantana (F3) apresentaram a menor. Filmes de amido e gelatina (F4) apresentam maior valor de RMT e elongação, enquanto os filmes de gelatina e goma xantana (F6) apresentaram o menor valor de elongação (Tabela 2).

Tabela 2. Resultados de permeabilidade ao vapor de água (PVA), resistência máxima à tração (RMT) e elongação dos filmes

\begin{tabular}{|c|c|c|c|c|c|c|}
\hline 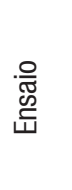 & 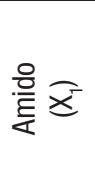 & 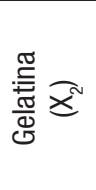 & 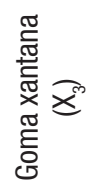 & 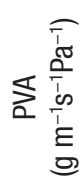 & 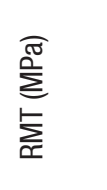 & 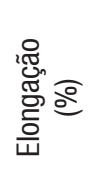 \\
\hline F1 & 1 & 0 & 0 & 3,71 & 53,90 & 10,70 \\
\hline F2 & 0 & 1 & 0 & 3,72 & 80,41 & 15,38 \\
\hline F3 & 0 & 0 & 1 & 5,95 & 18,42 & 4,61 \\
\hline F4 & 0,50 & 0.50 & 0 & 3,90 & 108,46 & 15,58 \\
\hline F5 & 0,50 & 0 & 0,50 & 4,98 & 65,23 & 9,52 \\
\hline F6 & 0 & 0,50 & 0,50 & 5,21 & 15,04 & 3,33 \\
\hline F7 & 0,33 & 0,33 & 0,33 & 4,18 & 59,12 & 8,62 \\
\hline
\end{tabular}

F1 (amido puro), F2 (gelatina pura), F3 (xantana pura), F4 (mistura binária amido e gelatina), F5 (mistura binária amido e xantana), F6 (mistura binária gelatina e xantana) e F7 (mistura ternária amido, gelatina e xantana)

Observando-se os modelos matemáticos gerados para a RMT e para a elongação, pode-se perceber que os efeitos dos três componentes da mistura foram significativos e positivos (Tabela 3 ), no entanto, os efeitos do amido $\left(X_{1}\right)$ e da gelatina $\left(X_{2}\right)$ foram mais importantes, e isso se observa na Figura 2 , em que se verifica que as regiões com máximos de gelatina e amido levaram ao aumento tanto da RMT, quanto da elongação.
Tabela 3. Modelos matemáticos ${ }^{\mathrm{a}}$ obtidos para a permeabilidade ao vapor de água (PVA), resistência máxima à tração (RMT) e elongação dos filmes

\begin{tabular}{cccc}
\hline Coeficientes & PVA & RMT & Elongação \\
\hline 1 & $3,75^{\star}$ & $54,43^{\star}$ & $10,74^{\star \star}$ \\
2 & 3,76 & $80,94^{\star \star}$ & $15,42^{\star \star}$ \\
3 & $5,99^{\star \star}$ & 18,95 & $4,65^{\star}$ \\
12 & $-0,07$ & 154,59 & 9,33 \\
13 & $-0,21$ & 105,65 & 6,63 \\
23 & 0,69 & $-148,13$ & $-27,49$ \\
P (modelo) & 0,3632 & 0,1279 & $0,0691^{\star}$ \\
$\mathrm{R}^{2}$ & 0,95 & 0,99 & 0,99 \\
\hline
\end{tabular}

$\mathrm{a}_{-}{ }_{1}=$ amido: $_{2}=$ gelatina: ${ }_{3}=$ goma xantana, ${ }^{* *} \mathrm{p} \leq 0,05 ;{ }^{*} \mathrm{p} \leq 0,1$.

Os resultados do grau de intumescimento (Gl) das amostras estão apresentados na Tabela 4. Os filmes de amido puro ( $\mathrm{F} 1)$ e a mistura ternária entre amido, gelatina e goma xantana (F7) apresentaram os menores valores de $\mathrm{Gl}$ em praticamente todos os tempos. Já a formulação de goma xantana pura (F3) e a mistura entre 0 amido e a goma xantana (F5) apresentaram os maiores valores de Gl, seguidos pela formulação F4 (mistura binária entre amido e gelatina), que apresentou elevados valores de Gl (Tabela 4).

Os altos valores de Gl nos filmes com a goma xantana podem ser explicados pela alta capacidade de ligação deste polissacarídeo com a água. A goma xantana classifica-se como um heteropolissacarídeo, e a unidade básica repetidora da molécula de xantana é um pentassacarídeo formado por duas unidades de D-glicose, duas unidades de D-manose e uma unidade de ácido glucurônico na proporção molar 2,8; 2,0; 2,0, além de grupos piruvato e acetil. As cadeias laterais da xantana apresentam interação eletrostática entre si e com a cadeia principal, e essas interações são responsáveis por importantes propriedades do polissacarídeo, das quais a mais importante para a exploração industrial é a capacidade de formar soluções aquosas de alta viscosidade em baixas concentrações (0,5 - 1,0\%) (GARCÍA-OCHOA et al., 2010). 

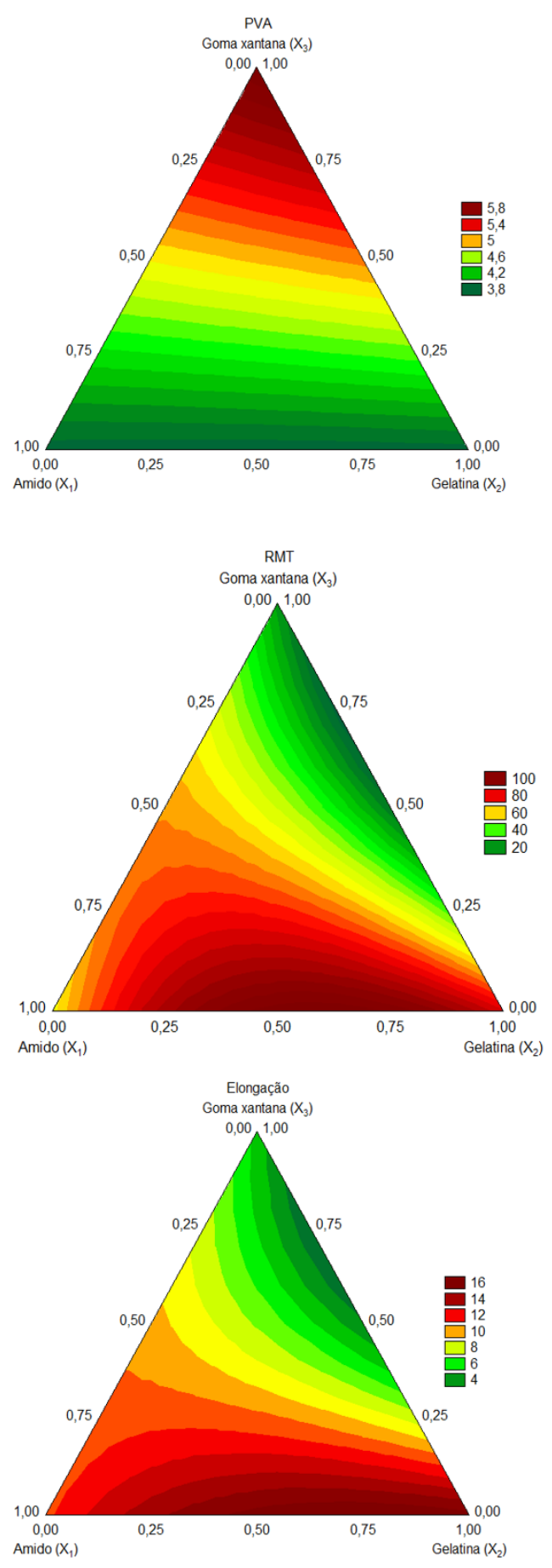

Figura 2. Gráficos de contorno com os efeitos do amido $\left(X_{1}\right)$, gelatina $\left(X_{2}\right)$ e goma xantana $\left(X_{3}\right)$ sobre a permeabilidade ao vapor de água (PVA), resistência máxima à tração (RMT) e elongação dos filmes
A solubilidade em água é um paramêtro importante para caracterização dos filmes biodegradáveis, quanto maior a porcentagem de amostra solúvel melhor para a aplicação como forma farmacêutica orodispersível. Em relação à porcentagem de solubilidade em diferentes tempos, as amostras F4 (mistura binária entre amido e gelatina), F6 (mistura binária entre gelatina e goma xantana) e F7 (mistura ternária entre os 03 biopolímeros) foram as amostras que apresentaram os maiores valores, enquanto que as amostras de amido puro (F1), xantana pura (F3) e a mistura binária entre amido e goma xantana (F5) apresentaram os menores valores de solubilidade (Tabela 5).

\section{CONCLUSÃo}

Os polímeros utilizados e o modo de preparo empregado mostraram-se satisfatórios no preparo dos filmes. Os filmes produzidos apresentaram boa aparência e homogeneidade. Em função dos resultados observados, os filmes orodispersíveis preparados com a mistura binária de amido e gelatina apresentaram a melhor combinação de resultados de solubilidade e as melhores propriedades mecânicas, configurando-se como uma alternativa promissora para 0 seu estudo como veículo para a liberação de princípios ativos na cavidade bucal.

\section{AGRADECIMENTOS}

Os autores agradecem ao CNPq - Brasil, pelo suporte financeiro; ao Laboratório de Microscopia e Microanálises (LMEM) - Universidade Estadual de Londrina. 
Tabela 4. Resultados do grau de intumescimento dos filmes em diferentes tempos

\begin{tabular}{|c|c|c|c|c|c|}
\hline \multirow{2}{*}{ Ensaio } & \multicolumn{5}{|c|}{ Grau de intumescimento $(\mathrm{g} / \mathrm{g})$} \\
\hline & $30 \mathrm{~s}$ & $1 \mathrm{~min}$ & $2 \min$ & $3 \mathrm{~min}$ & $4 \mathrm{~min}$ \\
\hline $\mathrm{F} 1$ & $2,21 \pm 0,26 d$ & $3,05 \pm 0,21 d$ & $4,78 \pm 0,47 \mathrm{c}$ & $4,18 \pm 0,16 c$ & $4,28 \pm 0,50 c$ \\
\hline $\mathrm{F} 2$ & $2,47 \pm 1,13 d$ & $5,30 \pm 1,45 a$ & $3,94 \pm 0,73 d$ & $4,79 \pm 1,01 \mathrm{C}$ & $4,81 \pm 2,31 \mathrm{c}$ \\
\hline F3 & $3,88 \pm 0,22 b$ & $4,23 \pm 0,85 b$ & $5,56 \pm 1,50 b$ & $8,50 \pm 0,46 a$ & $7,99 \pm 0,12 a$ \\
\hline $\mathrm{F} 4$ & $3,34 \pm 0,71 b$ & $5,77 \pm 0,94 a$ & $5,62 \pm 0,18 b$ & $5,86 \pm 0,69 b$ & $5,77 \pm 0,58 b$ \\
\hline $\mathrm{F} 5$ & $2,87 \pm 0,46 c$ & $3,71 \pm 0,49 c$ & $6,88 \pm 0,72 a$ & $7,17 \pm 1,18 \mathrm{a}$ & $7,00 \pm 1,11 a$ \\
\hline F6 & $5,22 \pm 0,99 a$ & $4,74 \pm 1,16 b$ & $4,38 \pm 0,39 c$ & $4,69 \pm 0,74 \mathrm{c}$ & $4,59 \pm 1,29 c$ \\
\hline F7 & $2,15 \pm 0,86 d$ & $3,98 \pm 0,44 b, c$ & $3,92 \pm 0,08 \mathrm{c}, \mathrm{d}$ & $4,36 \pm 1,54 \mathrm{c}$ & $5,22 \pm 1,26 b, c$ \\
\hline
\end{tabular}

Letras diferentes na mesma linha indicam diferença significativa pelo teste de Tukey (p£0,05). F1 (amido puro), F2 (gelatina pura), F3 (xantana pura), F4 (mistura binária amido e gelatina), F5 (mistura binária amido e xantana), F6 (mistura binária gelatina e xantana) e F7 (mistura ternária amido, gelatina e xantana).

Tabela 5. Porcentagem de solubilidade dos filmes em diferentes tempos das amostras

\begin{tabular}{|c|c|c|c|c|c|}
\hline \multirow{2}{*}{ Ensaio } & \multicolumn{5}{|c|}{ Solubilidade (\%) } \\
\hline & $30 \mathrm{~s}$ & $1 \mathrm{~min}$ & $2 \min$ & $3 \mathrm{~min}$ & $4 \min$ \\
\hline F1 & $16,61 \pm 5,15^{b}$ & $14,79 \pm 2,37^{b}$ & $14,48 \pm 7,55^{c}$ & $17,54 \pm 7,11^{b, c}$ & $14,99 \pm 1,07^{b, c}$ \\
\hline F2 & $13,71 \pm 3,04^{b, c}$ & $17,46 \pm 6,92^{b}$ & $19,62 \pm 7,09^{c}$ & $18,56 \pm 6,46^{b}$ & $25,12 \pm 2,95^{\mathrm{a}}$ \\
\hline F3 & $10,70 \pm 1,84^{c}$ & $10,05 \pm 6,56^{c}$ & $10,13 \pm 2,65^{d}$ & $11,11 \pm 2,27^{c}$ & $16,85 \pm 2,06^{b}$ \\
\hline F4 & $19,70 \pm 4,52^{\mathrm{a}}$ & $16,21 \pm 3,18^{b}$ & $27,19 \pm 6,72^{\mathrm{a}}$ & $26,52 \pm 0,15^{\mathrm{a}}$ & $26,29 \pm 3,74^{a}$ \\
\hline F5 & $14,55 \pm 0,05^{b, c}$ & $16,13 \pm 3,39^{b}$ & $14,22 \pm 8,38^{c}$ & $14,86 \pm 7,04^{b, c}$ & $13,91 \pm 1,36^{c}$ \\
\hline F6 & $18,69 \pm 3,38^{a}$ & $24,42 \pm 3,29^{a}$ & $23,77 \pm 3,52^{\mathrm{a}}$ & $24,42 \pm 1,77^{\mathrm{a}}$ & $28,04 \pm 1,39^{a}$ \\
\hline F7 & $21,86 \pm 2,44^{a}$ & $25,23 \pm 4,66 a$ & $26,33 \pm 6,035^{a}$ & $26,79 \pm 2,82^{\mathrm{a}}$ & $25,76 \pm 1,33^{a}$ \\
\hline
\end{tabular}

Letras diferentes na mesma linha indicam diferença significativa pelo teste de Tukey (p£0,05). F1 (amido puro), F2 (gelatina pura), F3 (xantana pura), F4 (mistura binária amido e gelatina), F5 (mistura binária amido e xantana), F6 (mistura binária gelatina e xantana) e F7 (mistura ternária amido, gelatina e xantana) 


\section{REFERÊNCIAS}

AMERICAN SOCIETY FOR TESTING AND MATERIALS. Standard test methods for water vapor transmission of material - E-96-00. In: ANNUAL book of ASTM standards. Philadelphia: ASTM, 2000a.

AMERICAN SOCIETY FOR TESTING AND MATERIALS. Standard test methods for seal strength of flexible barrier materials - ASTM F882-91. In: ANNUAL book of ASTM standards. Philadelphia: ASTM, 2000b.

BORGES, A. F.; SILVA, C.; COELHO J. F. J.; SIMÕES, S. Oral films: current status and future perspectives I galenical development and quality attributes. Journal of Controlled Release, v. 206, p. 1-19, may 2015.

CASTRO, P. M.; FONTE, P.; OLIVEIRA, A.; MADUREIRA, A. R.; SARMENTO, B.; PINTADO, M. E. Optimization of two biopolymer-based oral films for the delivery of bioactive molecules. Materials Science and Engineering C, v. 76, p. 171-180, 2017.

GALIANO, F.; BRICENO, K.; MARIANO, T.; MOLINO, A.; CHRISTENSEN, K. V.; FIGOLI, A. Advances in biopolymer-based membrane preparation and applications. Journal of Menbrane Science, v. 564, p. 562-586, 2018.

GARCÍA-OCHOA, F.; SANTOS, V. E.; CASAS, J. A.; GÓMEZ, E. Xanthan gum: production, recovery, and properties. Biotechnology Advances, v. 18, p. 549579, 2010.

LUCENA, C. A. A.; COSTA, S. C.; ELEAMEN, G. R. A.; MENDANÇA, E. A. M.; OLIVEIRA, E. E. Desenvolvimento de biofilmes a base de xilana e xilana/gelatina para produção de embalagens biodegradáveis. 2017. Dissertação (Mestrado em Ciências Farmacêuticas) - Universidade Estadual da Paraíba, Campina Grande, 2017.

MALI, S.; SAKANAKA, L. S.; YAMASHITA, F.;
GROSSMAN, M. V. E. Water sorption and mechanical properties of cassava starch films and their relation to plasticizing effect. Carbohydrate Polymers, v. 60, p. 283-289, 2005.

MANDEEP, K.; RANA, A. C.; NIMRATA, S. Fast dissolving films: an innovative drug delivery system. International Journal of Pharmaceutical Research \& Allied Sciences, v. 2, p. 14-24, 2013.

PALLAVI, P.; SHRIVASTAVA S. K. Fast Dissolving oral films: an innovative drug delivery system. International Journal of Science and Research, v. 3, p. 412-105, 2014.

PARK, S. B.; LIH, E.; PARK, K. S.; JOUNG, Y. K.; HAN, D. K. Biopolymer-based functional composites for medical applications. Progress in Polymer Science, v. 68, p. $77-105,2017$.

PATEL, K.; SONI, S.; PATEL, R.; PANDYA, V.; BHARADIA, $P$. Mouth dissolving film: a review. International Journal for Pharmaceutical Research Scholars, v. 1, p. 154-163, 2012.

PRAJAPATI, B. G.; RATNAKAR, N. A Review on recent patents on fast dissolving drug delivery system. International Journal of PharmTech Research, v. 1, p. 790-798, 2009.

ROMI0, A. P. et al. Encapsulação do carvacrol em filmes comestíveis de amido de mandioca e gelatina. Embrapa Instrumentação, p. 213-216, 21 nov. 2017.

SU, K.; WANG, C. Recent advances in the use of gelatin in biomedical research. Biotechnology Letters, v. 37, n. 11, p. 2139-2145, nov. 2015.

SUEIRO, A. C. Desenvolvimento de filmes orodispersíveis à base de amido de mandioca, proteína de soja, gelatina e pululana. 2018. Dissertação (Mestrado em Biotecnologia) - 
Departamento de Bioquímica e Biotecnologia,

Universidade Estadual de Londrina, Londrina, 2018.

TEDESCO, M. P.; MONACO-LOURENÇO, C.

A.; CARVALHO, R. A. Gelatin/hydroxypropyl

methylcellulose matrices - polymer interactions

approach for oral desintegrating films. Materials

Science and Engineering C, v. 69, p. 668-674,

2016.

VERCELHEZE, A. E.; MARIM. B. M.; OLIVEIRA, A. L. M.; MALI, S. Development of biodegradable coatings for maize seeds and their application for Azospirilum brasilense immobilization. Applied Microbiology and Biotechnology, v. 103, n. 5, p. 2193-2203, mar. 2019 .

VILLANOVA, J. C. 0.; OREFICE, R. L.; CUNHA, A. S.

Aplicações farmacêuticas de polímeros. Polímeros, v. 20, n. 1, p. 51-64, 2010.

Recebido em: 26/04/2019

Aceito em: 05/06/2019 